\title{
Central nervous system exposure of next generation quinoline methanols is reduced relative to mefloquine after intravenous dosing in mice
}

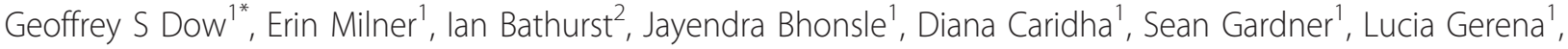 \\ Michael Kozar ${ }^{1}$, Charlotte Lanteri ${ }^{1}$, Anne Mannila ${ }^{3}$, William McCalmont ${ }^{1}$, Jay Moon ${ }^{1}$, Kevin D Read ${ }^{4}$, \\ Suzanne Norval ${ }^{4}$, Norma Roncal', David M Shackleford ${ }^{3}$, Jason Sousa', Jessica Steuten ${ }^{3}$, Karen L White ${ }^{3}$, \\ Qiang Zeng ${ }^{1}$ and Susan A Charman ${ }^{3}$
}

\begin{abstract}
Background: The clinical use of mefloquine (MQ) has declined due to dose-related neurological events. Next generation quinoline methanols (NGQMs) that do not accumulate in the central nervous system (CNS) to the same extent may have utility. In this study, CNS levels of NGQMs relative to MQ were measured and an early lead chemotype was identified for further optimization.

Experimental design: The plasma and brain levels of MQ and twenty five, 4-position modified NGQMs were determined using LCMS/MS at $5 \mathrm{~min}, 1,6$ and $24 \mathrm{~h}$ after IV administration $(5 \mathrm{mg} / \mathrm{kg})$ to male FVB mice. Fraction unbound in brain tissue homogenate was assessed in vitro using equilibrium dialysis and this was then used to calculate brain-unbound concentration from the measured brain total concentration. A five-fold reduction CNS levels relative to mefloquine was considered acceptable. Additional pharmacological properties such as permeability and potency were determined.

Results: The maximum brain (whole/free) concentrations of MQ were 1807/4.9 ng/g. Maximum whole brain concentrations of NGQMs were 23 - $21546 \mathrm{ng} / \mathrm{g}$. Maximum free brain concentrations were 0.5 to $267 \mathrm{ng} / \mathrm{g}$. Seven (28\%) and two (8\%) compounds exhibited acceptable whole and free brain concentrations, respectively. Optimization of maximum free brain levels, IC90s (as a measure or potency) and residual plasma concentrations at $24 \mathrm{~h}$ (as a surrogate for half-life) in the same molecule may be feasible since they were not correlated. Diamine quinoline methanols were the most promising lead compounds.
\end{abstract}

Conclusion: Reduction of CNS levels of NGQMs relative to mefloquine may be feasible. Optimization of this property together with potency and long half-life may be feasible amongst diamine quinoline methanols.

\section{Background}

The Walter Reed Army Institute of Research and collaborators are attempting to identify next generation quinoline methanols for intermittent preventive treatment (IPT) of malaria. IPT is the prevention of morbidity or mortality due to malaria through the intermittent administration of a single dose treatment of a drug at full therapeutic doses to asymptomatic, otherwise healthy infants

\footnotetext{
* Correspondence: geoffrey.dow@us.army.mil

'Division of Experimental Therapeutics, Walter Reed Army Institute of Research, Silver Spring, MD, USA

Full list of author information is available at the end of the article
}

(IPTi), pregnant women (IPTp) and travelers (IPTt) [1-3]. Drugs for IPTx indications and prophylaxis should ideally exhibit a long half-life, be very well-tolerated and safe in pregnancy. Mefloquine exhibits two of these characteristics, but will likely not find use as an IPT drug because of the adverse CNS events observed at the treatment level doses [4] that may be required for IPT. However, this would presumably not be an issue for next generation analogs of mefloquine without such a liability. Mefloquine accumulates in the CNS and has multiple CNS targets (see discussion in earlier papers $[5,6]$ ). The goal is to identify a lead compound for IPT, based on a

\section{() Biomed Central}


mefloquine scaffold, for which accumulation into the CNS is substantially reduced. Such a compound should have an improved CNS safety profile relative to mefloquine.

In an earlier study, it was demonstrated that non-piperidine quinoline methanols, in which the piperidine group of mefloquine was replaced with a diamine side chain, were metabolically stable, exhibited reasonable potency against Plasmodium falciparum in vitro and were less permeable across MDCK cell monolayers than their monamine counterparts $[7,8]$. That study did not attempt to address whether reductions in brain concentration relative to mefloquine could be achieved in vivo. This was the goal of the present study, in which approximately 25 compounds from our original library were resynthesized, and brain and plasma concentrations were measured over $24 \mathrm{~h}$ in mice after i.v. dosing. Plasma concentrations were measured to generate a preliminary indication of half-life, brain concentrations to assess potential exposure relative to mefloquine, and IC90s to assess intrinsic activity against P. falciparum.

The dose-limiting CNS side effects of mefloquine at the full therapeutic doses required for IPT include dizziness, incoordination, anxiety and sleeplessness [9]. These common side effects are largely absent at the weekly dose of mefloquine, which is five-fold lower than the treatment dose [10]. Therefore, assuming linearity of mefloquine pharmacokinetics in humans, it makes sense that, assuming no change in affinity for the putative CNS receptors of mefloquine, a five-fold reduction in CNS total drug levels would be the minimum requirement to reasonably expect an improvement in the therapeutic index of a NGQM delivering efficacy at blood exposure equivalent to mefloquine. However, as reported elsewhere $[7,8]$, the lipophilicity of diamine quinoline methanols and other early lead chemotypes is lower than mefloquine. Conceivably this might alter non-specific binding in the brain leading to an increase in the free brain concentration of the drug. Since we do not know the relevant clinical CNS target(s) of mefloquine and the importance of the total and free brain concentration in relation to adverse effects, it is important that reduction relative to mefloquine be assessed with respect to both parameters.

\section{Methods}

Library synthesis and physicochemical properties

Racemic mefloquine and its (+) and (-) erythro enantiomers were obtained from the WRAIR chemical inventory system. A sub-library of 25 next generation quinoline methanols was synthesized as described in our earlier papers $[7,8]$. Compounds were almost all rule of 5 (RO5) compliant [11] and designed to encompass the widest feasible range of physiochemical properties (see Table 1). All physiochemical properties were calculated
Table 1 Ranges of physiochemical properties of quinoline methanols investigated in this study

\begin{tabular}{cc}
\hline Parameter & Range \\
\hline MW & $324-475$ \\
CLogD (PP) & $1.0-5.2$ \\
CLogD $(A C D)$ & $-2.3-3.1$ \\
PSA $\left(\AA^{2}\right)$ & $36-104$ \\
LogP & $0.4-3.7$ \\
\# FRBs & $3-11$ \\
\# HBDs & $1-5$ \\
\# HBAs & $3-7$ \\
\hline
\end{tabular}

using ACD (Version 10, ACD Labs, Toronto, Canada) except for $\log \mathrm{D}(\mathrm{pH}$ 7.4) which was determined using Pipeline Pilot. (Version 6.1, Accelrys, San Diego, California). These properties have been defined in detail elsewhere [12], and numerically represent the size (MW), lipophilicity $(\log \mathrm{D}$ and $\log \mathrm{P})$, molecular flexibility (FRBs) and H-bonding capacity (HBDs and HBAs) of a compound, all of which impact biological properties important in drug development. The importance of these properties in drug discovery generally and to next generation quinoline methanols more specifically is discussed elsewhere [8]. Given that the focus of this study was on brain penetration, the sub-library of compounds was selected such that a wide range of brain levels was expected based on their physiochemical properties.

\section{Animals, dosing and collection of samples}

All in vivo studies were conducted in accordance with protocols approved by the Victorian College of Pharmacy, Animal Ethics Committee. Male, six week old FVB mice were obtained from the Animals Resources Center (Perth, Western Australia) and provided with food and water ad libitum. Mice were administered a 50 $\mu \mathrm{L}$ bolus injection of mefloquine, its enantiomers or one of twenty five quinoline methanol compounds by tail vein injection (dosing solution concentration $2.8 \mathrm{mg} / \mathrm{ml}$ ) for a nominal dose of $5 \mathrm{mg} / \mathrm{kg}$. The compounds were prepared in 5\% DMSO in 5\% glucose solution buffered to a $\mathrm{pH}$ of 3-5 using citrate or acetate as appropriate based on an assessment of the lipophilicity and pKa of the compound. At $5 \mathrm{~min}, 1 \mathrm{~h}, 6 \mathrm{~h}$ or $24 \mathrm{~h}$ post-dosing, mice were anaesthetized with inhaled isoflurane (3\%) approximately 3-4 minutes prior to blood and brain harvest. Blood was collected by cardiac puncture from groups of 2-3 mice and the whole brain was removed. The whole brains were placed into preweighed polypropylene vials and snap frozen prior to storage along with the plasma samples at $-20^{\circ} \mathrm{C}$ until analysis. On the day of analysis, the whole brain was homogenized in three parts of aqueous solution containing 0.1 M EDTA and $0.4 \mathrm{~g} / \mathrm{L} \mathrm{KF}$ (inhibitors of hydrolytic enzymes to reduce 
the potential for ex vivo tissue-mediated degradation of the test compounds). Calibration standards were prepared by spiking blank brain homogenate and blank plasma with the test compound. Both samples and standards were processed by adding acetonitrile (to precipitate proteins) and centrifuged to separate the supernatant for analysis. An aliquot of the supernatant from each brain homogenate and plasma samples were analysed by UPLC/MS/MS on either a Quattro Premier XE, a Quattro Ultima Pt or a Xevo XT Mass Spectrometer (Waters Corporation, USA).

\section{Determination of unbound brain fractions by equilibrium dialysis and free brain levels}

The unbound fraction of each analog in mouse brain homogenate was determined in vitro using equilibrium dialysis according to methodology reported previously [13]. In brief, a 96 well equilibrium dialysis apparatus was used to determine the unbound fraction in brain homogenate for each compound (HT Dialysis LLC, Gales Ferry, CT). Membranes (12-14 kDA cut-off) were conditioned in deionized water for 60 minutes, followed by conditioning in 80:20 deionized water:ethanol for 20 minutes, and then rinsed in artificial cerebrospinal fluid (CSF) before use. Mouse brain was removed from the freezer and allowed to thaw on the day of experiment. Thawed brain tissue was then homogenized with artificial CSF to a final composition of 1:2 brain:artificial CSF using a Covaris S2 (K Biosciences, Hoddesdon, UK). Diluted brain homogenate was then spiked with the test compound (10 ug/g), and $150 \mu \mathrm{L}$ aliquots ( $\mathrm{n}=6$ replicate determinations) loaded into the 96-well equilibrium dialysis plate. Dialysis vs artificial CSF $(150 \mu \mathrm{L})$ was carried out for 5 hours in a temperature controlled incubator at $c a .37^{\circ} \mathrm{C}$ (Barworld Scientific Ltd, UK) using an orbital microplate shaker at 125 revolutions/minute (Barworld scientific Ltd, UK). At the end of the incubation period, aliquots of brain homogenate or artificial CSF were transferred to micronic tubes (Micronic B.V., the Netherlands) and the composition in each tube balanced with control fluid, such that the volume of artificial CSF to brain was the same. Sample extraction was performed by the addition of $400 \mu \mathrm{L}$ of acetonitrile containing an appropriate internal standard. Samples were allowed to mix for 1 minute and then centrifuged at $3000 \mathrm{rpm}$ in 96well blocks for 15 minutes (Allegra X12-R, Beckman Coulter, USA). All samples were then analysed by UPLC/MS/MS on a Quattro Premier XE Mass Spectrometer (Waters Corporation, USA). The unbound fraction in brain was determined as the ratio of the peak area in artificial CSF to that in brain, with correction for dilution factor according to eq.1 [14].

$$
\text { Undiluted } f_{u}=\frac{1 / D}{\left(\left(1 / f_{u, \text { apparent }}\right)-1\right)+1 / D}
$$

where $D=$ dilution factor in brain homogenate and $f_{u}$, apparent is the measured free fraction of diluted brain homogenate.

Free brain levels were determined from measured whole brain concentrations and the undiluted $f_{u}$ as follows:

Free Brain Concentration $=$ Whole Brain Concentration $\times$ Undiluted $f_{u}$

\section{Determination of apparent permeability across MDCK cell layers}

Permeability was determined by Absorption Systems (Exton, PA). MDR1-MDCK cell monolayers were grown to confluence on collagen-coated, microporous, polycarbonate membranes in 12-well Costar Transwell plates. Data were considered valid for a specific assay plate if TEER values were $<1400 \Omega . \mathrm{cm}^{2}$, the $\mathrm{P}_{\text {app }}$ of propanolol was between $10-30 \times 10^{-6} \mathrm{~cm} / \mathrm{s}$ and the $\mathrm{P}_{\text {app }}$ of atenolol was $<0.5 \times 10^{-6} \mathrm{~cm} / \mathrm{s}$. The permeability assay buffer was Hanks Balanced Salt Solution containing $10 \mathrm{mM}$ HEPES and $15 \mathrm{mM}$ glucose at a $\mathrm{pH}$ of 7.4. A known $\mathrm{p}-$ glycoprotein inhibitor, cyclosporin A (CSA), was also added to the assay buffer at $10 \mu \mathrm{M}$. This was omitted for amphoteric compounds. Bovine serum albumin (1\%) was added to the receiver well. The dosing solution concentrations of the test compounds were $5.0 \mu \mathrm{M}$ in the assay buffer. All cell monolayers were first pre-incubated for 30 minutes with assay buffer. After 30 minutes, the buffer was removed, replaced with fresh buffer, and time was recorded as 0 . The addition of BSA, pre-incubation, and use of a longer incubation time were employed to mitigate potential low recovery or permeability that is sometimes observed for lipophilic or 'sticky' compounds. Cell monolayers were dosed on the apical side (A-to-B) or basolateral side (B-to-A) and incubated at $37^{\circ} \mathrm{C}$ with $5 \% \mathrm{CO} 2$ in a humidified incubator. After two hours, aliquots were taken from the receiver chambers. Samples were taken from the donor chamber at 0 and 2 hours. Each determination was performed in duplicate. The lucifer yellow flux was also measured for each monolayer to assess monolayer integrity during the flux period. All samples were assayed by LC/MS/MS using electrospray ionization.

Apparent permeability in the apical (A-B direction), Papp $_{\mathrm{A}-\mathrm{B}}$, and percent recovery are reported in the supplementary Information. Apparent permeability is a measure of the rate of transport across the cell monolayer. Percent recovery refers to the amount of compound recoverable at the end of the assay. Low recovery 
may indicate non-specific binding to assay plates, instability or accumulation in the cell pellet. In the case of mefloquine, relatively low recovery is likely a consequence of accumulation in cell membranes $[15,16]$ rather than non-specific binding.

The apparent permeability, Papp, and percent recovery were calculated as follows:

$$
\begin{aligned}
& \text { Papp }=(d \mathrm{Cr} / d \mathrm{t}) \times \mathrm{Vr} /(\mathrm{A} \times \mathrm{C} 0) \\
& \text { Percent Recovery }=100 \times\left(\left(\mathrm{Vr} \times \mathrm{Cr}^{\text {final }}\right)+\left(\mathrm{Vd} \times \mathrm{Cd}^{\text {final }}\right)\right) /(\mathrm{Vd} \times \mathrm{CN})
\end{aligned}
$$

where,

$d \mathrm{Cr} / d \mathrm{t}$ is the slope of the cumulative concentration in the receiver compartment versus time in $\mu \mathrm{M} \mathrm{s}^{-1}$.

$\mathrm{Vr}$ is the volume of the receiver compartment in $\mathrm{cm}^{3}$.

$\mathrm{Vd}$ is the volume of the donor compartment in $\mathrm{cm}^{3}$.

$\mathrm{A}$ is the area of the cell monolayer $\left(1.13 \mathrm{~cm}^{2}\right.$ for 12 well Transwell).

$\mathrm{C} 0$ is the measured concentration of the donor chamber at time 0 in $\mu \mathrm{M}$.

$\mathrm{CN}$ is the nominal concentration of the dosing solution in $\mu \mathrm{M}$.

$\mathrm{Cr}^{\mathrm{final}}$ is the cumulative receiver concentration in $\mu \mathrm{M}$ at the end of the incubation period.

$\mathrm{Cd}^{\text {final }}$ is the concentration of the donor in $\mu \mathrm{M}$ at the end of the incubation period.

\section{Plasmodium falciparum susceptibility assays}

The in vitro activities of quinoline methanols against P. falciparum strains W2, D6, TM91C235, and TM90C2A were evaluated using the traditional labeled hypoxanthine assay of Desjardins et al [17] as modified by Milhous et al [18]. These four P. falciparum strains were selected since they have various levels of resistance to conventional anti-malarials. W2 is chloroquine resistant and mefloquine sensitive, D6 is chloroquine sensitive but naturally less susceptible to mefloquine, TM91C235 is resistant to mefloquine, chloroquine, and pyrimethamine as is TM90C2A, however this latter parasite is a two $p f m d r 1$ copy strain (pfmdr1 amplification has been associated with clinical mefloquine resistance). Mefloquine is routinely screened in these assays to ensure the validity. Historical values for mefloquine against all the strains are reported elsewhere [8]. The focus of this study was the mefloquine-sensitive strain W2, however values for the other strains for all the compounds are reported in the Supplementary Information.

\section{Data analysis}

Prism graphing software was used to graph key datasets in order to facilitate visual inspection for possible correlations amongst parameters of interest followed by statistical confirmation if needed. For graphical analysis of IC90 data, IC90s were assumed to be equivalent to the maximum concentrations tested where they were insufficiently potent to be determined. Our brain uptake studies yielded plasma concentration data for four time points. This was insufficient for the purposes of determining half-lives in most instances. As a surrogate for half-life, we determined the \% residual plasma concentrations at $24 \mathrm{~h}$ versus concentrations at $5 \mathrm{~min}$. Where the measured plasma concentration was below the analytical lower limit of quantitation (LLQ), an arbitrary value of $1 \mathrm{ng} / \mathrm{ml}$ was assigned for the purposes of analysis. For analysis of MDCK permeability data versus maximum brain concentration, we did not adjust Papp values on the basis of \% recovery. However, percent recovery values are included in the Supplementary Information. The probability of a diamine exhibiting the desired free brain concentration, IC90s and residual plasma concentration was calculated by multiplying the marginal probabilities of each of these events since they appeared to be independent. The $+/-95 \%$ confidence limits for the probability estimate was calculated using the formula: $+/-95 \% \mathrm{CIs}=1.96 * \mathrm{SQRT}\left(\mathrm{P}^{*}(1-\mathrm{P}) / \mathrm{n}\right)$ where $\mathrm{P}$ is probability of success and SQRT is the square root of the result of the function in brackets.

\section{Results}

Twenty-five quinoline methanols were successfully resynthesized or obtained from the WRAIR chemical inventory system. Their structures are depicted in Figure 1. Physicochemical properties, in vitro susceptibility data, brain tissue binding data, brain and plasma concentrations and MDCK screening data are summarized in Additional file 1. The range of physicochemical properties encompassed by the mini-library of compounds is summarized in Table 1.

The maximum whole and free brain concentrations of the quinoline methanols are summarized in Figures 2A and $2 \mathrm{~B}$, respectively. The maximum whole and free brain concentrations of racemic mefloquine were 1807 and $4.9 \mathrm{ng} / \mathrm{g}$. For the (+) and (-) mefloquine enantiomers, these values were (whole/free) 1159/2.5 and 2249/ $5.2 \mathrm{ng} / \mathrm{g}$, respectively. The lowest whole brain concentration was $23 \mathrm{ng} / \mathrm{g}$ (WR318470) and the lowest free brain concentration was $0.45 \mathrm{ng} / \mathrm{g}$ (WR319535). Of the 25 novel compounds, 7 (28\%) and $2(8 \%)$ passed the minimum acceptable five-fold reduction in whole and free brain levels, respectively.

Pharmacological data for four important parameters were examined for evidence of possible correlations in order to justify assumptions regarding probability estimates and to assess the feasibility of the optimization of desirable traits in a single molecule. There was no evidence of correlations amongst the following parameters: 


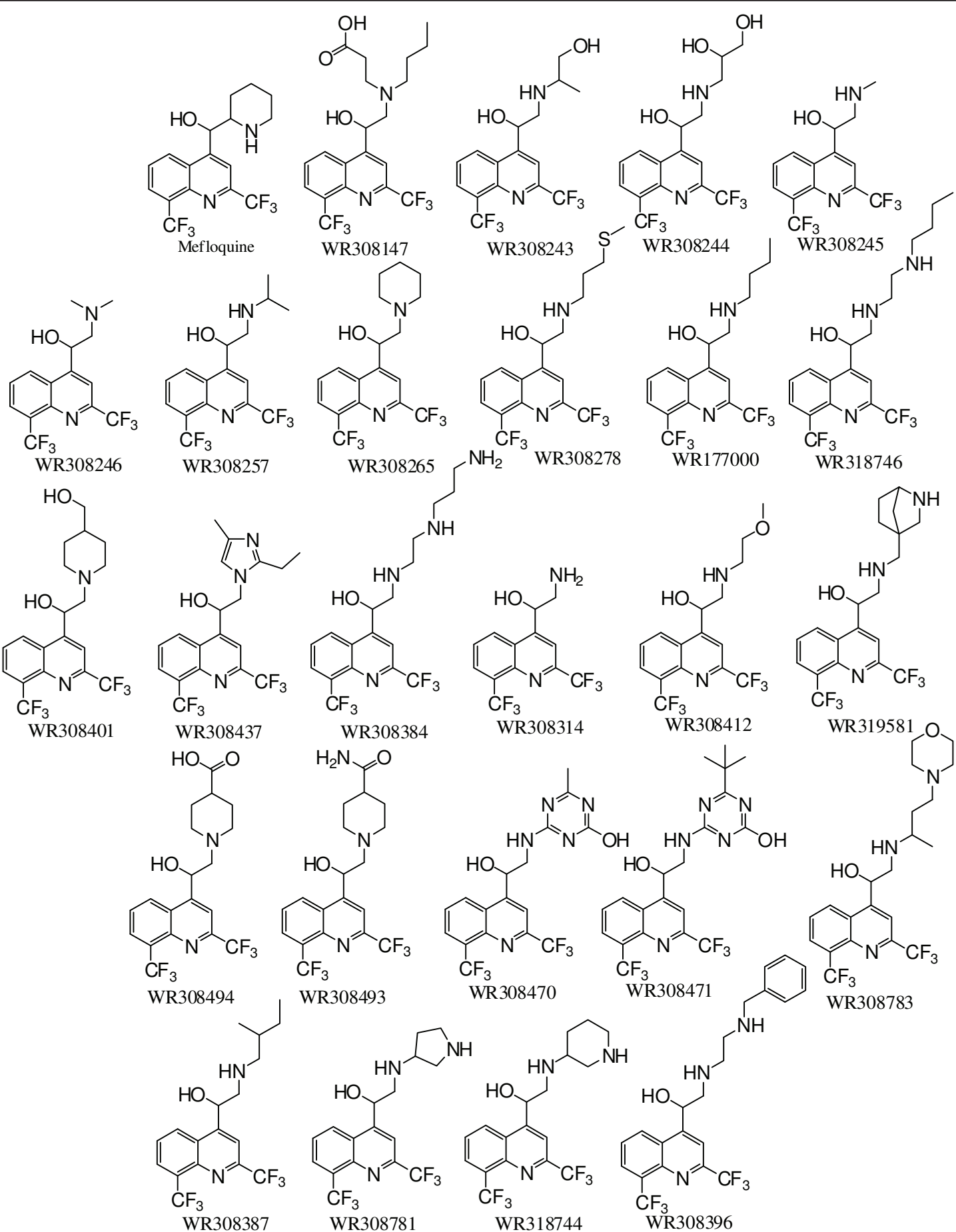

Figure 1 Structures of quinoline methanols investigated in this study. The structure of racemic mefloquine is indicated. Its two diasteromers were also investigated in this study.

residual plasma concentrations at $24 \mathrm{~h}$, maximum free brain concentration and IC90s (Figures 3A-C). This suggests that (i) their probability distributions may be independent and (ii) they may be optimizable in the same molecule. There was an apparent linear relationship evident between maximum whole and free brain concentrations (Figure 3D), that was confirmed mathematically $\left(\mathrm{r}^{2}=0.89, P<0.0001\right.$, linear regression). This suggests that these variables are not independent and that $89 \%$ of the variability in free brain concentrations 


\section{A}

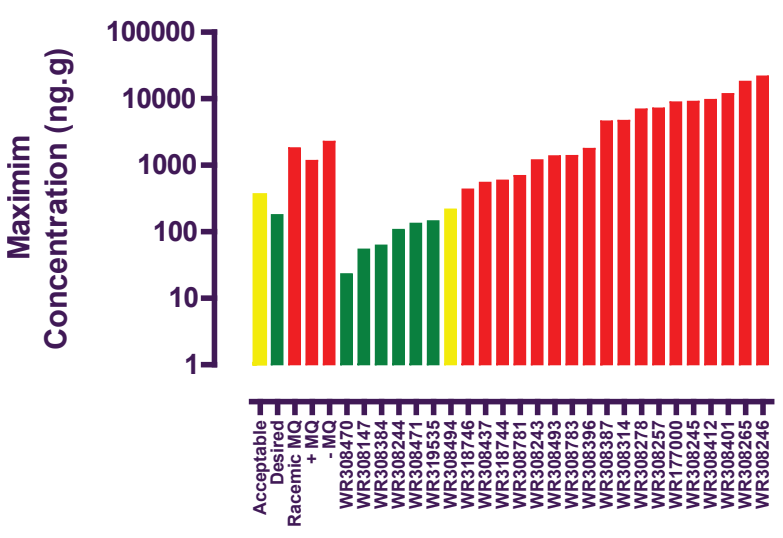

B

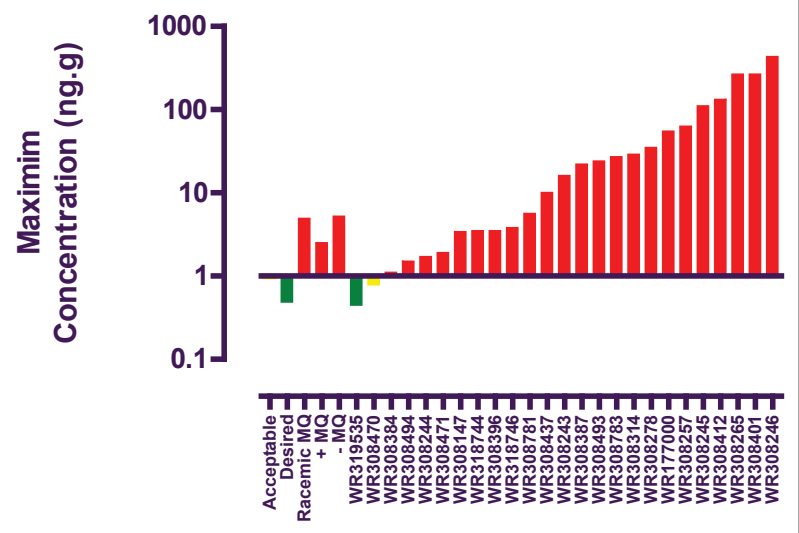

Figure 2 Maximum whole (A) and free (B) brain concentrations for racemic mefloquine, + erythro mefloquine, (-) erythro mefloquine and 25 novel quinoline methanols after IV dosing to male FVB mice $(5 \mathrm{mg} / \mathbf{k g})$. Compounds are color-coded green, yellow or red based on whether they exhibited the desired (> tenfold lower than racemic mefloquine), acceptable (> five-fold lower than racemic mefloquine) or unacceptable brain levels, respectively.

can be explained by variability in whole brain concentrations alone. There was no qualitative evidence of a simple linear correlation between IC90 and maximum whole brain concentration (Figure 3E). However, the lack of data in the lower left quadrant suggests more caution in making the assumption of independence between these two variables. There was no evidence of correlation between residual drug concentrations and maximum whole brain concentration (Figure 3F).

One of the most promising compounds was WR319535 (Figures 1, 2 and 4). This compound exhibited maximum whole and free brain concentrations of 145 and $0.45 \mathrm{ng} / \mathrm{g}$, respectively. Significant drug levels remained at $24 \mathrm{~h}$ postdosing (Figure 4) compared to many of the other compounds investigated (Supplementary Information) which may imply a long half-life. Unfortunately, the compound exhibited only modest potency. Four related diamine quinoline methanols (Figure 5) were also evaluated. When these were considered together, 1 in 5 exhibited appropriate potency, 1 in 5 exhibited appropriate whole brain levels, and 1 in 5 exhibited appropriate free brain levels relative to mefloquine. All of these compounds were represented in the group of 9 non-piperidine compounds with residual plasma concentrations $>1 \%$ of the observed maximum value. This suggests they represent the sub-chemotype with the greatest propensity for a long half-life. The probability (+/- 95\% confidence intervals) of such a molecule exhibiting the desired free brain levels, potency and residual plasma concentrations was estimated to be 4 (0-21)\% (Figure 5).

The apparent permeability (A-B) of quinoline methanols was used to categorize them as having either higher (if Papp was $>7.4 \times 10^{-6} \mathrm{~cm} / \mathrm{s}$ ) or lower (if Papp was < $7.4 \times 10^{-6} \mathrm{~cm} / \mathrm{s}$ ) maximum brain concentrations than mefloquine in vivo (Table 2). Compounds with apparent permeability values of $<7.4 \times 10^{-6} \mathrm{~cm} / \mathrm{s}$ exhibited lower brain concentrations than mefloquine in vivo in $100 \%$ of cases. Compounds with apparent permeability $>7.4 \times$ $10^{-6} \mathrm{~cm} / \mathrm{s}$ exhibited maximum brain concentrations higher than mefloquine in $71 \%$ of cases (Table 2). Thus, is it is likely that an MDCK permeability acceptability threshold of $<7.4 \times 10^{-6} \mathrm{~cm} / \mathrm{s}$ would exclude most future compounds for which maximum brain concentrations are higher than mefloquine.

\section{Discussion}

The focus of the WRAIR discovery program with next generation quinoline methanols has been to reduce partitioning into the CNS since the relevant clinical target or targets of mefloquine adverse events are not known [19]. The incidence of the most common adverse CNS effects of mefloquine is reduced substantially when the dose is lowered five-fold from the treatment to the weekly prophylaxis level. Presumably this is also associated with a five-fold decline in CNS levels as well. Consequently, at least a five-fold reduction in whole and free brain levels relative to mefloquine at the efficacious dose is probably the minimum requirement. In this study we have shown that at least a ten-fold reduction in both parameters is feasible through replacement of the piperidine ring of mefloquine with various other four-position substituents. The prospect of such a compound being better tolerated than mefloquine is, therefore, a possibility.

While a reduction in maximum brain concentration should reduce the incidence of CNS associated adverse events, optimizing this property alone will not be sufficient for a new quinoline methanol anti-malarial. The putative late lead compound must also have a 


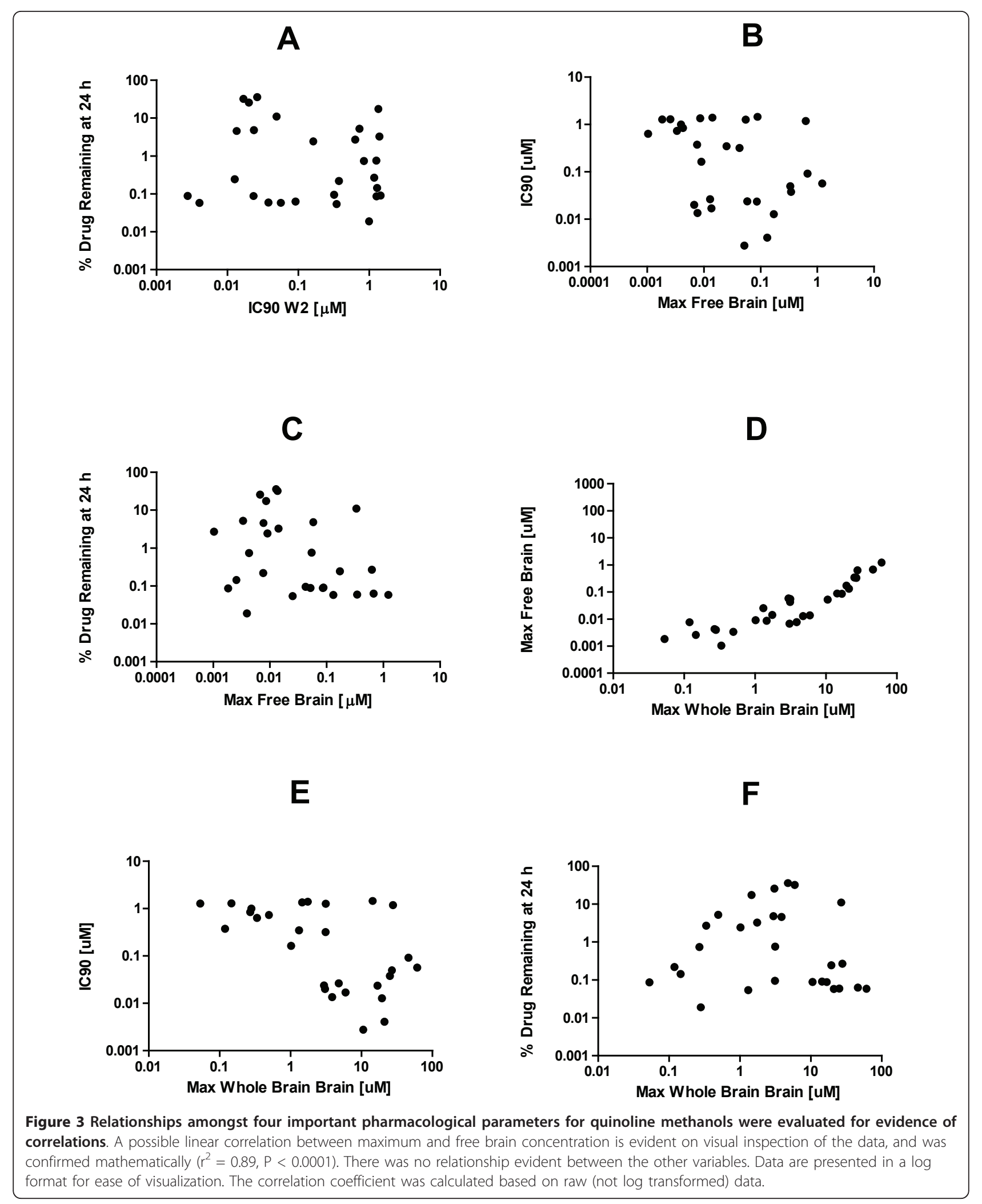




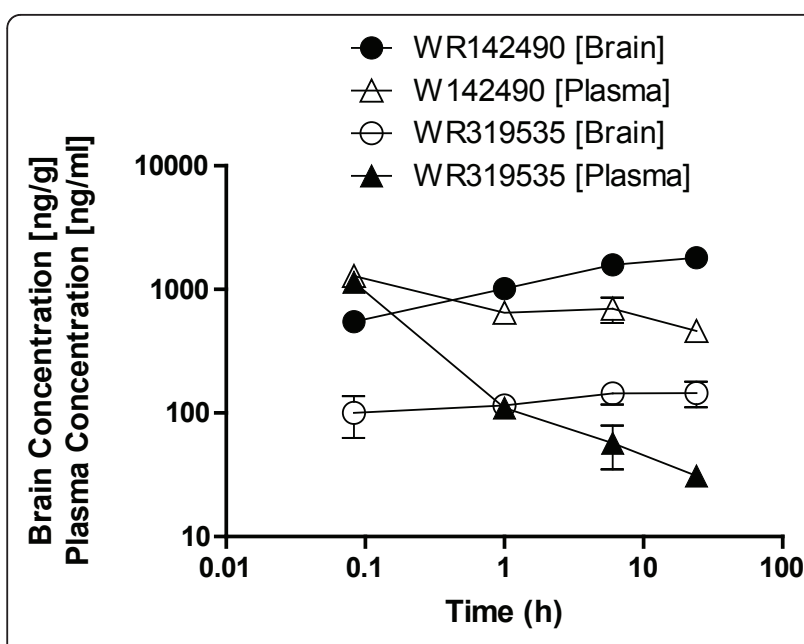

Figure 4 Brain and plasma concentrations of racemic mefloquine and the diamine WR319581 after IV dosing $(5 \mathrm{mg} / \mathrm{kg})$ to male FVB mice.

sufficiently long half-life and intrinsic efficacy to be useful if administered as a single dose. Therefore, the goal is to balance these three potentially competing pharmacological properties in the same molecule. There is an opportunity to evaluate a subset of the larger 4-position library using surrogates of these endpoints. Maximum free and whole brain concentrations represent surrogates of CNS safety if they are sufficiently reduced relative to mefloquine at the therapeutic dose. Whilst the plasma concentration-time data generated are insufficient to calculate pharmacokinetic parameters directly in most instances, the proportion of drug remaining at $24 \mathrm{~h}$ relative to the 5 minute time point is probably a reasonable surrogate of half-life, or at least persistence in plasma. In vitro activity (IC90) can be utilized as a surrogate for intrinsic activity.

There are no compounds amongst this sub-library that meet all the requirements in terms of potency, low brain levels and residual plasma concentrations at $24 \mathrm{~h}$ (see Additional file 1) suggesting that further optimization is required. The ideal chemotype around which to anchor a future lead optimization program would be one in which a balance between all these three properties is achievable. The most active compounds were generally those with aliphatic, ether or thio ether side chains (WR308245, WR308246, WR308257, WR308265, WR177000, WR308387, WR308278 and WR308412). However, these compounds exhibited much higher brain concentrations (free and whole) than mefloquine, and in most instances, $24 \mathrm{~h}$ residual plasma concentrations were $<1 \%$ of those at $5 \mathrm{~min}$, indicating a likely short half-life relative to mefloquine. The exception to this general trend was the diamine WR308396. As might be expected, all compounds with lower whole and free brain concentrations than mefloquine had a greater number of H-bond donors and/or acceptors than mefloquine. These compounds comprised a diverse array of structures including alcohols, acids, triazines, substituted cyclic imidazoles and diamines. However, with the exceptions of the diamines WR319581 and WR318746, these compounds exhibited a lack of potency (IC90 > $300 \mathrm{ng} / \mathrm{ml})$ or low residual plasma concentrations $(<1 \%$ at $24 \mathrm{~h}$ versus $5 \mathrm{~min}$ ). Of the 25 compounds that were not mefloquine or its enantiomers, only nine had residual plasma concentrations $>1 \%$ at $24 \mathrm{~h}$, and five of these were diamines. Consequently, a balance between these properties may be most likely obtained amongst diamine quinoline methanols.

Identifying a new lead compound with the requisite balance of potency, brain partitioning and half-life might not be feasible if it was true that positive trends in these characteristics were mutually exclusive, however this does not appear to be the case. Maximum free brain concentrations, IC90s, and residual plasma concentrations amongst quinoline methanols did not appear to be correlated (Figure 3A-C). Therefore, at the outset there is no reason to suspect that a balance between these properties is not achievable in a diamine quinoline methanol. There are no generic algorithms that can be used to objectively judge the probability of success of a specific lead optimization programme a priori. Thus one must extrapolate on a project-by-project basis with the available data. The lack of a general correlation between maximum free brain concentrations, residual plasma concentrations and IC90s suggests that their probability distributions may be independent. Thus, we estimated the probability of a diamine quinoline methanol possessing all these properties to be around $4 \%$. This value was obtained by multiplying the marginal probabilities of success for the individual parameters of interest (Figure 5). The confidence intervals are wide $(0-21 \%)$ which is not surprising given the small sample sizes.

The probability estimate discussed above was based on free brain concentrations being representative of CNS exposure. We have not made the same calculations for maximum whole brain concentrations, because the assumption of independence between this variable and IC90 may arguably be less justifiable. However, two lines of evidence support the notion that a compound with an acceptable maximum free brain concentrations will also have an acceptable maximum whole brain concentrations. First, whole and free brain concentrations in the broader group of quinoline methanols were strongly correlated. This suggests that they are also not independent, or put another way, that there is a strong conditional probability whole brain concentrations will be lower than mefloquine if this is the case for free brain concentrations. Second, in the same broader 
<smiles>OC(c1cc(C(F)(F)F)nc2c(C(F)(F)F)cccc12)C1CCCCN1</smiles>

Mefloquine

IC90 W2 = 10

Whole Brain Cmax $=1807$

Free Brain $\mathrm{Cmax}=4.9$

$24 \mathrm{~h} \%$ Remaining $=35$<smiles>OC(CNC1CCNC1)c1cc(C(F)(F)F)nc2c(C(F)(F)F)cccc12</smiles>

WR308781

IC90 W2 > 500

Whole Brain $\mathrm{Cmax}=689$

Free Brain $\mathrm{Cmax}=5.6$

$24 \mathrm{~h} \%$ Remaining $=3.3$<smiles>CCCCNCCNCC(O)c1cc(C(F)(F)F)nc2c(C(F)(F)F)cccc12</smiles>

WR 318746

IC90 W2 = 69

Whole Brain Cmax $=431$

Free Brain $\mathrm{Cmax}=3.8$

$24 \mathrm{~h} \%$ Remaining $=2.4$<smiles>OC(CNC1CCCNC1)c1cc(C(F)(F)F)nc2c(C(F)(F)F)cccc12</smiles>

WR3 18744

IC $90 \mathrm{~W} 2=>500$

Whole Brain $\mathrm{Cmax}=593$

Free Brain $\mathrm{Cmax}=3.5$

$24 \mathrm{~h} \%$ Remaining $=17$<smiles>OC(CNCCNCc1ccccc1)c1cc(C(F)(F)F)nc2c(C(F)(F)F)cccc12</smiles>

WR308396

IC90 W2 = 6

Whole Brain Cmax $=1768$

Free Brain $\mathrm{Cmax}=3.5$

$24 \mathrm{~h} \%$ Remaining $=4.6$<smiles>OC(CNCC12CCC(CC1)NC2)c1cc(C(F)(F)F)nc2c(C(F)(F)F)cccc12</smiles>

WR319535/581

IC90 W2 = 275

Whole Brain $\mathrm{Cmax}=145$

Free Brain $\mathrm{Cmax}=0.45$

$24 \mathrm{~h} \%$ Remaining $=2.7$

Figure 5 Structures, IC90s, maximum whole and free brain concentrations, and residual $24 \mathrm{~h}$ plasma concentrations of diamine quinoline methanols. IC90s are expressed in $\mathrm{ng} / \mathrm{ml}$ against the mefloquine-sensitive W2 strain of $P$. falciparum. Maximum whole and free brain concentrations in male FVB mice after iv dosing $(5 \mathrm{mg} / \mathrm{kg})$ are expressed in $\mathrm{ng} / \mathrm{g}$. Residual plasma concentrations at $24 \mathrm{~h}$ after iv dosing are expressed as a percentage relative to the 5 minute time point. One in 5 diamines (20\%, WR308396) had acceptable potency. One in 5 diamines had acceptable whole (20\%, WR319535) and free (20\%, WR319535) brain levels relative to mefloquine. WR319535 exhibited a half-life longer than mefloquine after oral dosing (data not shown). For this reason we have assumed that the residual plasma concentrations at $24 \mathrm{~h}$ of all (100\%) of the diamines may be indicative of long half-lives.

group of quinoline methanols it is also true that the proportion of compounds exhibiting the desired whole brain concentrations $(28 \%)$ was higher than the proportion of compounds exhibiting the desired free brain concentrations (8\%). Additional studies are required to determine whether these observations will hold for a larger sample of diamine quinoline methanols.

It is appropriate to execute a lead optimization programme if it can be done with a reasonable probability of success in a time frame of up to two years [20]. For

Table 2 Utility of MDCK permeability screen for ranking maximum brain concentrations of quinoline methanols relative to mefloquine.*

Compounds with Papp $<7.4 \times 10^{-6}$ Compounds with Papp $>7.4 \times 10^{-6}$ $\mathrm{cm} / \mathrm{s}$ $\mathrm{cm} / \mathrm{s}$

Compounds with lower maximum brain concentrations than 10

4

mefloquine

Compounds with higher maximum brain concentrations than

0

10 mefloquine

* Racemic mefloquine and isomers excluded 
planning purposes, it has been assumed the true probability of success for an individual compound lies somewhere between the lower confidence interval and $4 \%$. Since the synthetic route is amenable to the synthesis of 100 new compounds in 12-18 months, it is anticipated that up to four potential late lead molecules will be identified in that time frame. The assumed low probability of success with any individual compound requires an aggressive method of early triage. The permeability of a compound across MDCK cell monolayers appears to correctly categorize it relative to mefloquine in terms of its maximum brain concentration in most instances. It is, therefore, reasonable to use this assay together with more routine $P$. falciparum susceptibility assays to rapidly prioritize compounds for in vivo studies. It may be necessary to conduct in vivo studies on a selection of active, but permeable compounds triaged using this technique in order to control the type II error rate. A lead optimization campaign conducted broadly along these lines is now underway.

\section{Additional material}

Additional File 1: Structures, physiochemical properties and biological data for a collection of diamine quinoline methanols. This file contains the structures of 25 diamine quinoline methanols, racemic mefloquine and its isomers, their key physiochemical properties, and biological data from Pf in vitro screening, cytotoxicity screening, MDCK permeability studies, plasma and brain binding experiments, and brain and plasma concentration data after i.v. dosing in mice. The methods are described in the text of the manuscript.

\section{Acknowledgements}

This manuscript was reviewed by the Walter Reed Army Institute of Research and the U.S. Army Medical Research and Materiel Command, and there is no objection to its publication or dissemination. The opinions expressed herein are those of the authors and do not necessarily reflect the views or opinions of the Department of the Army and the Department of Defense. All animal experiments were conducted in compliance with the Animal Welfare Act and other federal statutes and regulations relating to animals and experiments involving animals and adhere to the principles stated in the Guide for the Care and Use of Laboratory Animals (National Academy Press, 1996).

We gratefully acknowledge Medicines for Malaria Venture and the United States Military Infectious Diseases Research Program for financial support. The MDCK permeability assays were performed under contract by Absorption Systems (Exton PA). We thank Meng Shi for statistical advice.

\begin{abstract}
Author details
'Division of Experimental Therapeutics, Walter Reed Army Institute of Research, Silver Spring, MD, USA. ${ }^{2}$ Medicines for Malaria Venture, International Center Cointrin, Route de Pre-Bois 20, PO Box 1826, CH-1215 Geneva 15, Switzerland. ${ }^{3}$ Centre for Drug Candidate Optimisation, Monash Institute of Pharmaceutical Sciences, Monash University, 381 Royal Parade, Parkville, VIC 3052, Australia. ${ }^{4}$ Division of Biological Chemistry and Drug Discovery, College of Life Sciences, University of Dundee, Sir James Black Centre, Dundee, DD1 5EH, Scotland, UK.
\end{abstract}

\section{Authors' contributions}

All authors made contributions to this study through past or current membership of the Next Generation Quinoline Methanol project team. The objective of this project team is to identify a development candidate from the quinoline methanol class for malaria prophylaxis and IPT. GSD, KR and SC conceived the general project study. GSD analysed the data and prepared the manuscript. EEM, WFM, SG and JM synthesized the analogs. NR and LG determined IC90s. DC determined LC50s. SN performed the equilibrium dialysis. JS, JSC, DS, KW, QZ and SC coordinated and/or executed the brain uptake experiments. JB determined the physiochemical properties. All authors read and approved the final manuscript.

\section{Competing interests}

The authors declare that they have no competing interests.

Received: 6 December 2010 Accepted: 6 June 2011

Published: 6 June 2011

\section{References}

1. Dow GS, Magill AJ, Ohrt C: Clinical development of new prophylactic antimalarial drugs after the 5th Amendment to the Declaration of Helsinki. Ther Clin Risk Manag 2008, 4:803-819.

2. Schellenberg D, Cisse B, Mendendez C: The IPTi Consortium: research for policy and action. Trends Parasitol 2006, 22:296-300.

3. Shanks GD, Magill AJ, Freedman DO, Keystone JS, Bradley DJ, Steffen R: Drug-free holidays: pre-travel versus during travel malaria chemoprophylaxis. Am J Trop Med Hyg 2007, 77:1-2.

4. Rendi-Wagner P, Noedl H, Wernsdorfer WH, Weidermann G, Mikolasek A, Kollaristch $\mathrm{H}$ : Unexpected frequency, duration and spectrum of adverse events after therapeutic dose of mefloquine in healthy adults. Acta Trop 2002, 81:167-173.

5. Caridha A, Yourick D, Cabezas M, Wolf L, Hudson TH, Dow GS: Mefloquineinduced disruption of calcium homeostasis in mammalian cells is similar to that induced by ionomycin. Antimicrob Agent Chemother 2008, 52:684-693.

6. Dow G, Bauman R, Caridha D, Cabezas M, Du F, Gomez-Lobo R, Park M, Smith K, Cannard K: Mefloquine induces dose-related neurological effects in a rat model. Antimicrob Agent Chemother 2006, 50:1045-1053.

7. Milner E, McCalmont W, Bhonsle J, Caridha C, Carroll D, Gardner S, Gerena L, Gettayacamin M, Lanteri C, Luong T, Melendez V, Moon J, Roncal N, Sousa J, Tangtaeng A, Wipf P, Dow GS: Structure-activity relationships amongst 4-position quinoline methanol antimalarials that inhibit the growth of drug sensitive and resistant strains of Plasmodium falciparum. Bioorg Med Chem Lett 2010, 20:1347-1351.

8. Milner E, McCalmont W, Bhonsle J, Caridha D, Cobar J, Gardner S, Gerena L, Goodine D, Lanteri C, Melendez V, Roncal N, Sousa J, Wipf P, Dow G: Antimalarial activity of a non-piperidine library of next-generation quinoline methanols. Malar J 2010, 9:51.

9. Rendi-Wagner PH, Noedl H, Wernsdorfer WH, Weidermann G, Mikolasek A, Kollaritsch H: Unexpected frequency, duration and spectrum of adverse events after therapeutic dose of mefloquine in healthy adults. Acta Trop 2002, 81:167-173.

10. Overbosch D, Schilthuis H, Bienzle U, Behrens RH, Kain KC, Clarke PD, Toovey S, Knobloch J, Nothdurft HD, Shaw D, Roskell NS, Chulay JD: Atovaquone-proguanil versus mefloquine for malaria prophylaxis in non-immune travelers: results from a randomized, double-blind study. Clin Infect Dis 2001, 33:1015-1021.

11. Lipinski CA: Drug-like properties and the causes of poor solubility and poor permeability. J Pharmacol Toxicol Methods 2000, 44:235-249.

12. Kerns EH, Di L: Drug-like properties: concepts, structure design and methods. Burlington, MA, Elsevier Inc; 2008, 35-100.

13. Summerfield SG, Read K, Begley DJ, Obradovic T, Hidalgo IJ, Coggon S, Lewis AV, Porter RA, Jeffrey P: Central nervous system drug disposition: The relationship between in situ brain permeability and brain free fraction. JPET 2007, 322:205-213.

14. Kalvass JC, Maurer TS: Influence of nonspecific brain and plasma binding on CNS exposure: implications for rational drug discovery. Biopharmaceutics Drug Disp 2002, 23:327-338.

15. Go ML, Ngiam TL: Thermodynamics of partitioning of the antimalarial drug mefloquine in phospholipid bilayers and bulk solvents. Chem Pharm Bull 1997, 45:2055-2060.

16. Zidovetzki R, Sherman IW, Attiya A, De Boeck H: A nuclear magnetic resonance study of the interactions of the antimalarials chloroquine, 
quinacrine, quinine and mefloquine with dipalmitoylphosphatidylcholine bilayers. Mol Biochem Parasitol 1989, 35:199-207.

17. Desjardins RE, Canfield CJ, Haynes JD, Chulay JD: Quantitative assessment of antimalarial activity in vitro by a semiautomated microdilution technique. Antimicrob Agents Chemother 1979, 16:710-718.

18. Milhous WK, Weatherly NF, Bowdre JH, Desjardins RE: In vitro activities of and mechanisms of resistance to antifol antimalarial drugs. Antimicrob Agents Chemother 1985, 27:525-530

19. Dow GS, Magill AJ: Kucer's Use of Antibiotics.Edited by: Grayson ML, Crowe SM, McCarthy JS, Mills J, Mouton JW, Norrby SR, Paterson DL and Pfaller MA. ASM Press, Washington DC; , Sixth 2008:2024-2035, "Mefloquine".

20. Paul MS, Mytelka DS, Dunwiddie CT, Persinger CC, Munos BH, Lindborg SR, Schacht AL: How to improve R\&D productivity: the pharmaceutical industry's grand challenge. Nat Rev Drug Discovery 2010, 9:203-214.

doi:10.1186/1475-2875-10-150

Cite this article as: Dow et al: Central nervous system exposure of next generation quinoline methanols is reduced relative to mefloquine after intravenous dosing in mice. Malaria Journal 2011 10:150.

\section{Submit your next manuscript to BioMed Central} and take full advantage of:

- Convenient online submission

- Thorough peer review

- No space constraints or color figure charges

- Immediate publication on acceptance

- Inclusion in PubMed, CAS, Scopus and Google Scholar

- Research which is freely available for redistribution

Submit your manuscript at www.biomedcentral.com/submit 